\title{
Cooperación académica entre el sector productivo y las universidades: Una evaluación basada en las prácticas estudiantiles
}

\author{
Édixon Chacón-Guerrero* ${ }^{a *}$, Rolando Eslava-Zapata ${ }^{\mathrm{b}}$, Genny Chacón-Lobo $^{\mathrm{c}}$, Hilário \\ Alonso Gonzalez-Júnior ${ }^{d}$, Mariana del Valle Buitrago-Rodríguez ${ }^{\mathrm{e}}$ \\ aDoctor en Psicología, Universidad de los Andes (ID), bDoctor en Administración \\ de Organizaciones, Universidad de los Andes (D), 'Magíster en Administración. \\ Universidad de los Andes (ID. dAsistente de profesor, Universidad de los Andes. (ID \\ eProfesor en el Departamento de Ciencias Administrativas, de Mercantil e Instituciones \\ Financieras, Universidad de Los Andes Táchira, Venezuela. (ID
}

\footnotetext{
Forma de citar: : Chacón, E., Eslava, R., Chacón, G., Gonzalez, H. A. \& Buitrago, M. (2017). Cooperación académica entre el sector productivo y las universidades: Una evaluación basada en las prácticas estudiantiles. Perspectivas, 2(2). 17-31.
}

Recibido: febrero 06 de 2017

Aceptado: mayo 26 de 2017

\section{Palabras clave \\ Cooperación académica, pasantías, universidad, sector productivo regional}

Resumen: La presente investigación describe la cooperación académica que se produce entre el Sector Productivo Regional y la Universidad Nacional Experimental Politécnica de la Fuerza Armada Nacional (UNEFA) - Táchira - Venezuela, en el marco de las pasantías profesionales de los estudiantes de final de carrera. El estudio es de tipo descriptivo, con un diseño de campo, a través del cual se recolectó la información directamente de la realidad estudiada. La población estuvo conformada por 368 estudiantes en el proceso de pasantías en UNEFA Táchira, 29 docentes tutores y 291 organizaciones con convenio con la UNEFA Táchira. De las poblaciones de estudiantes y organizaciones, se extrajeron muestras representativas a través de la fórmula para población finita. También se realizó un estudio censal con los tutores académicos. Para la recolección de la información pertinente, se diseñaron tres

*Autor para correspondencia

edixonch@yahoo.com 
Keywords

Academic cooperation, internships, university, regional productive sector

\section{Palavras chave}

Cooperação acadêmica, estágios, universidades, setor produtivo regional instrumentos: un cuestionario dirigido a los estudiantes, un cuestionario que fue aplicado a los docentes tutores y un cuestionario dirigido a las empresas. De manera general, los resultados evidencian una escasa comunicación entre las organizaciones del sector productivo regional y la UNEFA Táchira, planes académicos poco ajustados a las realidades laborales actuales y poca integración entre el sector productivo y la universidad.

\section{Academic cooperation between the productive sector and universities: an evaluation based on student practices}

Abstract :This research describes the academic cooperation between the Regional Productive Sector and the National Experimental University of the Armed Forces (UNEFA) - Táchira - Venezuela, concerning the work experience placements of students in their final semester. The study is descriptive in nature, with a defined subject area, from which the information was directly collected from the reality under study. The population was composed of 368 UNEFA students with an internship contract, 29 tutors and 291 organizations under an agreement with UNEFA Táchira. From the student populations and organizations, representative samples were extracted through the finite population correction formula. A census study was also carried out with the academic tutors. For the collection of relevant information, three materials were designed: a questionnaire addressed to students, another to tutors and the final to the companies. In general, the results showed limited communication between the organizations of the regional productive sector and the UNEFA Táchira, the academic plans did not fulfill the current working realities and there is little integration between the productive sector and the university.

\section{Cooperação acadêmica entre o setor produtivo e as universidades: uma avaliação baseada em práticas estudantis}

Resumo: Esta pesquisa descreve a cooperação acadêmica que ocorre entre o Setor Produtivo Regional e a Universidade Experimental Politécnica Nacional das Forças Armadas Nacionais (UNEFA) - Táchira - Venezuela, no âmbito dos estágios profissionais dos alunos do último ano. O estudo é de tipo descritivo, com um projeto de campo, através do qual a informação foi coletada diretamente da realidade estudada. A população era composta por 368 alunos no processo de estágio na UNEFA Táchira, 29 professores e 291 organizações com acordo com a UNEFA Táchira. Das populações e organizações estudantis, amostras 
representativas foram extraídas através da fórmula para a população finita. Um estudo do censo também foi realizado com os tutores acadêmicos. Para a recolha da informação pertinente, foram concebidos três instrumentos: um questionário dirigido aos alunos, um questionário que foi aplicado aos professores dos tutores e um questionário dirigido às empresas. Em geral, os resultados mostram pouca comunicação entre as organizações do setor produtivo regional e UNEFA Táchira, planos acadêmicos pouco ajustados às atuais realidades trabalhistas e pouca integração entre o setor produtivo e a universidade.

\section{Introducción}

La cooperación académica en la educación superior se ha intensificado en los últimos años a través de distintas modalidades. Entre ellas se encuentran las pasantías universitarias de los estudiantes durante su última etapa de carrera. El mundo actual, caracterizado por fenómenos complejos como la globalización, hace necesaria la cooperación universitaria con el sector productivo (Dan, 2013), cooperación basada en la solidaridad entre las partes a fin de facilitar la movilidad de estudiantes, la concreción de convenios de cooperación y, en general, fomentar el mejoramiento de la calidad de la enseñanza en las universidades.

El proceso de cooperación académica debe ser adecuadamente gestionado a través de una estrategia global de cooperación que cubra los programas académicos institucionales y ofrezca oportunidades de desarrollo a los estudiantes (Morales Rubiano, Sanabria Rangel, Plata Pacheco y Ninco Hernández, 2015). Para ello se requiere un trabajo en conjunto que acerque la universidad con el sector productivo a fin de reducir la brecha entre la generación y la transferencia de conocimientos. El conocimiento obtenido con las pasantías universitarias está adquiriendo cada día un gran valor en la sociedad del conocimiento (De La Vega y Arakaki, 2011), sobre todo, por la experiencia que la realidad laboral supone para la formación, aprendizaje y desarrollo profesional (Rikap, 2012).

La gestión de las pasantías ocupa un lugar importante en el proceso universitario, considerando el desarrollo del conocimiento y el mejoramiento de las capacidades de los estudiantes que luego se convertirán en un factor de éxito institucional. Las pasantías, en efecto, permiten integrar la información con la experiencia práctica, que se transforma en la preparación de las capacidades de los estudiantes para la acción y crean un escenario propicio para que los estudiantes pongan en práctica los conocimientos adquiridos a lo largo de sus estudios (Corti, Oliva y De la Cruz, 2015).

La presente investigación tiene por finalidad describir el fortalecimiento de la cooperación académica entre la Universidad Nacional Experimental Politécnica de la Fuerza Armada Bolivariana (UNEFA) Núcleo Táchira, y el sector productivo regional durante el proceso de las pasantías universitarias. Para identificar los factores internos y externos que obstaculizan una eficaz cooperación académica se destaca la importancia del proceso relacionado con las pasantías universitarias y la vinculación académica existente entre la UNEFA, Núcleo Táchira, y el sector productivo regional.

\section{Cooperación académica y sector productivo}

La vinculación de las universidades con los sectores productivos adquiere cada día una mayor importancia, por lo que se hace necesario fortalecer los procesos que favorezcan los cambios que se experimentan en el mundo laboral debido a la globalización (Kneller, 2014). La educación superior participa de manera activa en el desarrollo 
social, económico y cultural de un país. De ahí que deba ser de interés institucional la transformación constante a fin de actualizarse y ofrecer programas académicos diversos y adecuados para la atención de las necesidades de las organizaciones en general Acevedo, J; González, E. (2017).

El sector productivo desempeña un rol importante en el proceso de transformación de la educación superior. Por tanto, los vínculos que relacionan el sector productivo y las Universidades deben generar acciones recíprocas de intercambio y beneficio (Kneller, 2014). Una de las alternativas consiste en las pasantías académicas que permiten relacionar al estudiante con la práctica profesional para que se familiaricen con los sectores productivos de la sociedad.

La internacionalización de los mercados hace necesario vincular aún más las instituciones de educación superior con los sectores productivos (Maculan y Carvalho de Mello, 2009). Esto supone que las Universidades debe modernizarse para hacer frente a la nueva realidad internacional que requiere de profesionales más competitivos y fomente la credibilidad de la institución en la formación de profesionales aptos para incorporarse al mercado laboral (Solanas, 2014).

Dentro de las universidades es fundamental la existencia de una oficina de Coordinación de Pasantías para que facilite la interrelación entre el sector productivo y las universidades, así como fomentar convenios de cooperación que vinculen al estudiante con el mercado profesional. Asimismo, la Coordinación puede asesorar a los estudiantes sobre los requerimientos y lugares donde puede desarrollar la pasantía. Sobra decir que todo este trabajo beneficia mutuamente a ambos sectores.

\section{Las pasantías universitarias}

Las pasantías universitarias son una forma de facilitar al estudiante su adaptación al medio laboral y permitirles ampliar su experiencia de la realidad práctica como complemento de los conocimientos teóricos adquiridos en las aulas de clases. Las pasantías ubican al alumno en el contexto del sector productivo actual para que sea capaz de relacionar la teoría y la práctica de manera que favorezcan su vida profesional (Allende Hernández, González Carella y Zanfrillo, 2010).

Las pasantías permiten aplicar los conocimientos teóricos a la resolución de problemas prácticos del sector productivo y la retroalimentación del proceso de enseñanza aprendizaje que, al final, termina por favorecer el desarrollo del futuro profesional (Nicholas, 2016). Es así como en un corto plazo, las instituciones de educación superior buscan relacionar los alumnos con los sectores productivos a fin de enriquecer las experiencias de la práctica (AlfiaGazizova, 2015).

Las universidades deben establecer políticas adecuadas de pasantías que permitan mejora la vinculación con el sector productivo (Rigsby, Addy, Dean yPolledo, 2013). Así, los estudiantes podrán mejorar sus capacidades potenciales para solucionar problemas. El proceso de pasantías es algo más que el producto de la relación entre la universidad con el sector productivo, pues supone también un esfuerzo conjunto entre las partes involucradas por aportar experiencias positivas al futuro profesional.

La pasantía es un mecanismo de vinculación de la universidad con el sector productivo para que el estudiante ponga en práctica conocimiento en beneficio de las empresas e las instituciones de educación superior involucradas (Renganathan, Abdul Karim y Li, 2012). Los resultados finales de las pasantías pueden ser muy satisfactorios para los alumnos en función de los logros alcanzados y para la Universidad, porque las acciones de este tipo mejoran el prestigio de la institución en el sector productivo. En términos generales, algunos beneficios de las pasantías pueden reflejarse en la consolidación de los conocimientos adquiridos por los alumnos en la Universidad (Templeton, Updyke y Bennett, 2012), la resolución de problemas prácticos inherentes a la realidad laboral y la confianza que se genera en el sector productivo por la educación proporcionada a los alumnos (SampaioCorrêa da Silva y Pereira Teixeira, 2013). 


\section{La educación y el sector productivo}

El sector productivo también juega un papel importante en el proceso de formación de los futuros profesionales (Grotta y McGrath, 2013). El vínculo que surge de la universidad con el sector productivo permite la movilidad de estudiantes a las empresas, lugar en el que pueden comprender los nuevos requerimiento y estándares de calidad demandados por el mercado laboral (García Palma, 2013).

El sector productivo tiene la posibilidad de establecer cronogramas de trabajo para los estudiantes y delimitar las acciones necesarias para llevar a cabo las tareas asignadas. En este sentido, de la relación entre el sector productivo y las universidades pueden surgir nuevas pautas para el mejoramiento de los programas de estudio de las carreras ofrecidas por las instituciones educativas, a fin de que se adapten a la nueva realidad de las organizaciones.

El sector productivo, siendo un demandante activo de profesionales, posee conocimientos claros de las habilidades y capacidades que las universidades deberían ofrecer a los estudiantes y puede impulsar experiencias en las empresas, promover el aprendizaje y formar profesionales críticos comprometidos con los retos formativos del mercado laboral.

El sector productivo también puede contribuir con recursos económicos (Weible, 2009), diseño de proyectos y acciones públicas que amplíen el abanico de posibilidades en apoyo a la educación, la formación y la capacitación de los estudiantes (Miranda, y Salto, 2012). La participación del sector productivo a la educación va más allá que la aceptación de un estudiante en la organización, pues también implica diseñar acciones orientadas a desarrollar políticas enfocadas a articular el trabajo con la universidad (Mgaya y Mbekomize, 2014).

Considerando los anteriores argumentos, en la presente investigación se pretende evaluar las distintas interrelaciones de cooperación académica que se dan entre el sector productivo y la universidad a través del proceso de prácticas profesionales (pasantías) con el fin de diagnosticar los puntos de encuentro y de mejora en aras del fortalecimiento de tales vínculos para la mejora de la formación profesional del estudiantado.

\section{Materiales y métodos}

Esta investigación se apoya en el paradigma cuantitativo $\mathrm{y}$, tomando en consideración el problema y los objetivos propuestos, se considera dentro del nivel descriptivo (Hernández Sampieri, Fernández Collado, y Baptista Lucio, 2006). En tal sentido, el propósito del trabajo ha sido describir la situación referida a la cooperación académica, en cuanto a las pasantías profesionales, que se da entre el Sector Productivo Regional y la Universidad Nacional Experimental Politécnica de la Fuerza Armada Nacional -Táchira - Venezuela (en adelante UNEFA- Táchira).

\subsection{Población y muestra}

El grupo de elementos a estudiarse en la presente investigación está conformado por (a) el conjunto de estudiantes de la UNEFA - Táchira del octavo semestre diurno de todas las especialidades, que actualmente se encuentran desarrollando sus pasantías profesionales, (b) los docentes tutores académicos y (c) los tutores organizacionales del periodo académico 2-2012 de las carreras de Ingeniera Civil, Ingeniería Eléctrica, Ingeniería en Sistemas, Administración, Gestión Municipal, Economía Social y Licenciatura de Turismo, organizaciones con las cuales existe, actualmente, un convenio de cooperación académica.

El cuestionario está dirigido a los coordinadores de pasantías de cada una de las organizaciones del sector productivo regional. El cuadro 1 muestra las poblaciones consideradas de acuerdo con información suministrada por la Coordinación de Pasantías de la UNEFA- Táchira. 
Cuadro 1

Población del estudio

\begin{tabular}{lc}
\hline \multicolumn{1}{c}{ Población } & Cantidad \\
\hline Estudiantes en proceso de pasantías & 368 \\
Docentes tutores académicos & 29 \\
Tutores organizacionales del periodo académico 2-2012 & 291 \\
\hline
\end{tabular}

En vista de que las poblaciones consideradas poseen amplias dimensiones (más de 100 elementos), se torna necesario calcular la muestra. Para el caso de los estudiantes y encargados de los pasantes en cada empresa se aplicó el muestreo aleatorio simple. Como parámetros se tomó el $95 \%$ de nivel de confianza y un máximo error del 5\%.
En relación con los docentes tutores, dadas sus características de accesibilidad, se recurrió al censo, el cual consistió en la aplicación del instrumento de medición (cuestionario) a todos ellos. En el cuadro 2 se presenta la muestra para cada una de las poblaciones.

Cuadro 2

Muestra seleccionada para el estudio

\begin{tabular}{lcc}
\hline \multicolumn{1}{c}{ Población/Estrato } & Cantidad & Muestra \\
\hline Estudiantes en proceso de pasantías & 368 & 188 \\
Docentes tutores académicos & 29 & 29 \\
Encargados de pasantías en las organizaciones con convenio de cooperación académica & 291 & 166 \\
\hline
\end{tabular}

Fuente: Autor, Proceso de investigación.

\subsection{Instrumentos de recolección de datos}

Para el estudio se diseñaron tres cuestionarios: (a) cuestionario que se aplicó a los estudiantes en proceso de pasantías, (b) cuestionario dirigido a docentes tutores y (c) cuestionario aplicado a las organizaciones con las cuales la UNEFA - Táchira que poseen un convenio de cooperación académica.

Para calcular la confiabilidad se utilizó el método de medidas de consistencia interna a través del Coeficiente de Alfa de Cronbach. En este caso, se aplicó una prueba piloto de cada cuestionario a estudiantes de la carrera de Licenciatura en Economía Social que cursaron la asignatura Matemática I durante el lapso 2-2010, en la UNEFA - Táchira y a docentes en el área de Ciencias Sociales y a un grupo de los encargados en las empresas, ninguno de los cuales forman parte de la población objeto de esta investigación.

Con la información suministrada se determinó el coeficiente de Alfa de Cronbach mediante la aplicación de la siguiente fórmula. En el caso del cuestionario para estudiantes el coeficiente obtenido fue de 0,83 ; lo cual significa que de 100 veces que este se aplique bajo las mismas condiciones, en aproximadamente 83 oportunidades el resultado será el mismo, lo cual indica que la confiabilidad es muy buena. Con relación al cuestionario para los docentes 
y de los representantes de las empresas, los cálculos arrojaron valores del Alfa de Cronbach superiores a 0,85 . El grado de confiabilidad del instrumento se considera altamente confiable, pues de cada 100 veces que se aplique el instrumento bajo las mismas condiciones, en aproximadamente 86 ocasiones se repetirán los resultados. Los valores obtenidos están cercanos a los parámetros de alta consistencia interna, quedando demostrada la confiabilidad de ambos instrumentos. Bajo estos parámetros de confiabilidad se aplicó el instrumento a la muestra seleccionada en el Ciclo Básico de Ingeniería. Los datos de la prueba piloto y de la muestra colecta fueron analizados aplicando técnicas de estadística descriptiva a través del programa SPSS versión 23.0

\section{Resultados y discusión}

\subsection{Análisis de las respuestas obtenidas a través del cuestionario aplicado a los tutores.}

El cuadro 3 relaciona los resultados de la distribución de frecuencias de los 13 ítems que conforman el cuestionario aplicado a tutores. El ítem 1 aborda la experiencia que proporcionan las pasantías al estudiante para su integración con éxito en el campo laboral.

Para el 58,62\% de los tutores encuestados, la experiencia que le proporcionan las pasantías al estudiante le permitirá integrarse con éxito en el campo laboral (ver cuadro 3).

Cuadro 3

Distribución por ítems del cuestionario aplicado a tutores

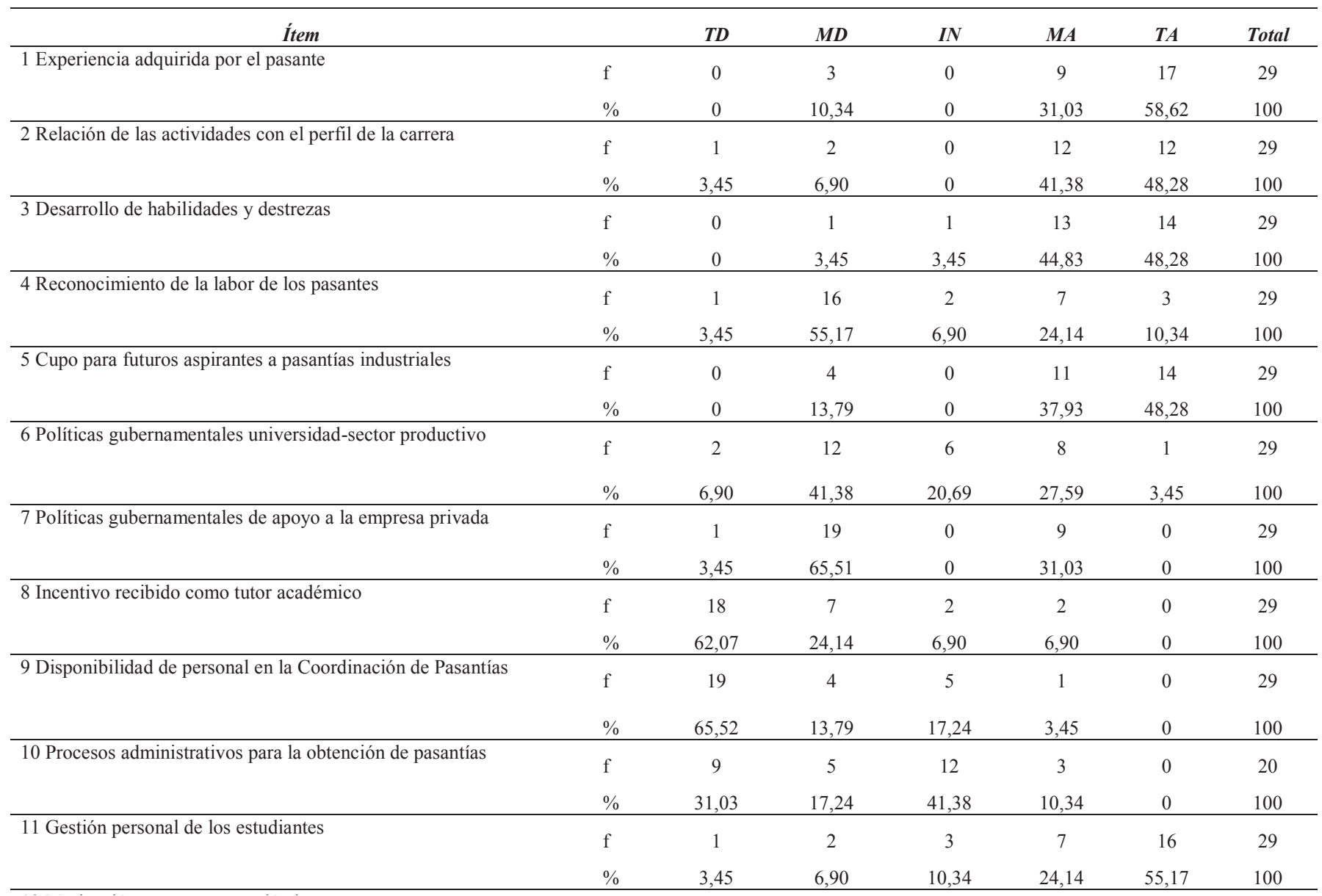




\begin{tabular}{|c|c|c|c|c|c|c|c|}
\hline 12 Motivación como tutor académico & $\mathrm{f}$ & 9 & 11 & 1 & 6 & 2 & 29 \\
\hline \multirow[t]{2}{*}{13 Disponibilidad de tiempo para las tutorías académicas } & $\mathrm{f}$ & 5 & 18 & 4 & 2 & 0 & 29 \\
\hline & $\%$ & 17,24 & 62,07 & 13,79 & 6,90 & 0 & 100 \\
\hline
\end{tabular}

Fuente: Autor, Proceso de investigación.

El ítem 2 relaciona las actividades que se desarrollan en las pasantías y que están directamente relacionadas con el perfil de la carrera de los estudiantes. En concordancia con las respuestas obtenidas en la pregunta anterior, los tutores expresan un alto nivel de acuerdo, equivalente al 89,66\%, en que las actividades que se desarrollan durante las pasantías están directamente relacionadas con el perfil de la carrera de los estudiantes $(48,28 \%$ totalmente de acuerdo y $41,38 \%$ medianamente de acuerdo). Al respecto, cabe destacar la importancia del rol que cumple el tutor académico en el seguimiento de las actividades desarrolladas por los pasantes en las empresas.

En relación a cómo las pasantías permiten al estudiante al estudiante de la UNEFA - Táchira desarrollar habilidades y destrezas necesarias para el desempeño profesional (ítem 3), según la opinión del $93,11 \%$ de los tutores encuestados resulta favorable (48,28 \% totalmente de acuerdo y 44,83 $\%$ medianamente de acuerdo).

El ítem 4 evalúa la opinión acerca de cómo la empresa reconoce la labor de los pasantes para un buen desempeño profesional. Se observa una importante proporción de los tutores encuestados, $55,17 \%$, está medianamente en desacuerdo con que la empresa reconoce la labor de los pasantes como altamente calificada para un buen desempeño profesional, seguido de un $24,14 \%$ que se encuentra medianamente de acuerdo con esta afirmación.

El ítem 5 está relacionado con si el desempeño de los estudiantes de la universidad asegura el cupo a futuros aspirantes a las pasantías industriales. Para un 48,28\% de los tutores, el desempeño de los estudiantes de la Universidad confirma el cupo a futuros aspirantes a las pasantías industriales, seguido de un $37,93 \%$ que está medianamente de acuerdo con esta afirmación.
La evaluación de los tutores sobre las políticas gubernamentales relacionadas con la vinculación sector productivo - universidad revela que $41,38 \%$ de los encuestados está medianamente en desacuerdo con que facilitan el proceso de obtención de pasantías por parte de los estudiantes. Por otra parte, en relación con la consideración de que las políticas gubernamentales relacionadas con el apoyo a la empresa privada facilitan el proceso de obtención de pasantías por parte de los estudiantes (ítem 7), 65,52\% de los se muestra medianamente en desacuerdo con el aserto.

En referencia a los incentivos que reciben los tutores (ítem 8) como recompensa a su labor como tutor académico, del cuadro 3 se desprende que una amplia proporción, del62,07\%, afirma estar totalmente en desacuerdo con que el incentivo que recibe recompensa su labor como tutor académico, seguido de un $24,14 \%$ que dice estar medianamente en desacuerdo. En referencia a la opinión que considera que la Coordinación de Pasantías de la UNEFA - Táchira posee el personal suficiente para un efectivo desempeño de sus funciones (ítem 9), el $65,52 \%$ de los tutores encuestados manifiesta estar totalmente en desacuerdo.

Sobre si los procesos administrativos en la universidad facilitan la obtención de pasantías, por parte de los estudiantes (Ítem 10 el 41,38\% se muestra, seguido de un $31,03 \%$ que está totalmente en desacuerdo. En este sentido, al preguntárseles si los estudiantes promueven personalmente la búsqueda de una opción para sus pasantías (Ítem 11) el $55,17 \%$ de los tutores encuestados está totalmente de acuerdo en que se trata de u procedimiento usual.

Al abordar a los tutores sobre su motivación en el desempeño de sus funciones como tutor académico (ítem 12), un $68,96 \%$ expresa sentir poca 
estimulación en sus labores $(37,93 \%$ medianamente en desacuerdo y $31,03 \%$ totalmente en desacuerdo).A este respecto, es preciso aclarar que los docentes de la UNEFA - Táchira no reciben remuneración adicional alguna como tutores académicos, puesto que se trata de funciones inherentes a su dedicación en la Universidad (dedicación exclusiva o contratados). En referencia a la disposición del tiempo necesario para el desarrollo de las actividades propias del tutor académico de pasantías (ítem 13), las respuestas emitidas por los tutores encuestados señalan que el $62,07 \%$ está medianamente en desacuerdo en cuanto al tiempo de que disponen para esta tarea, seguido de un $17,24 \%$ que opina estar totalmente en desacuerdo.

\subsection{Análisis de las respuestas obtenidas a través del cuestionario aplicado a las empresas}

El cuadro 4 muestra la distribución de frecuencias del cuestionario aplicado a las empresas. En referencia a si la empresa realiza algún tipo de donativo a la Universidad durante el transcurso de las pasantías industriales de los estudiantes (ítem 1) cabe destacar que ninguna de las consideradas para el estudio realiza aporte alguno. Lo expuesto es indicativo de que la cooperación académica entre el sector productivo local y la UNEFA - Táchira se limita a la posibilidad de que los estudiantes realicen sus pasantías profesionales en estas organizaciones.

Cuadro 4

Distribución por ítems del cuestionario aplicado a las empresas

\begin{tabular}{|c|c|c|c|c|c|}
\hline Ítem & & nunca & a veces & siempre & Total \\
\hline \multirow[t]{2}{*}{1 Donativo empresarial a la universidad } & $\mathrm{f}$ & 166.00 & 0.00 & 0.00 & 166 \\
\hline & $\%$ & 100.00 & 0.00 & 0.00 & 100 \\
\hline \multirow[t]{2}{*}{2 Patrocinio empresarial } & $\mathrm{f}$ & 166.00 & 0.00 & 0.00 & 166 \\
\hline & $\%$ & 100.00 & 0.00 & 0.00 & 100 \\
\hline \multirow[t]{2}{*}{3 Apoyo de proyectos comunes universidad - empresa } & $\mathrm{f}$ & 158.00 & 8.00 & 0.00 & 166 \\
\hline & $\%$ & 95,18 & 4,82 & 0.00 & 100 \\
\hline \multirow[t]{2}{*}{4 Reconocimiento a la labor destacada del pasante } & $\mathrm{f}$ & 56.00 & 76.00 & 34.00 & 166 \\
\hline & $\%$ & 33,73 & 45,78 & 20,48 & 100 \\
\hline \multirow[t]{2}{*}{5 Oportunidad de trabajo al pasante } & $\mathrm{f}$ & 86.00 & 75.00 & 5.00 & 166 \\
\hline & $\%$ & 51,81 & 45,18 & 3,01 & 100 \\
\hline \multirow[t]{2}{*}{6 Becas o créditos a los pasantes } & $\mathrm{f}$ & 164.00 & 1.00 & 1.00 & 166 \\
\hline & $\%$ & 98,80 & 0,60 & 0,60 & 100 \\
\hline \multirow[t]{2}{*}{7 Cupos para el desarrollo de pasantías industriales } & f & 39.00 & 84.00 & 43.00 & 166 \\
\hline & $\%$ & 23,49 & 50,60 & 25,90 & 100 \\
\hline \multirow[t]{2}{*}{8 Bonificación o pago a los pasantes } & $\mathrm{f}$ & 67.00 & 72.00 & 27.00 & 166 \\
\hline & $\%$ & 40,36 & 43,37 & 16,27 & 100 \\
\hline \multirow{2}{*}{9 Comunicación con la Coordinación de Pasantías } & $\mathrm{f}$ & 123.00 & 39.00 & 4.00 & 166 \\
\hline & $\%$ & 74,16 & 23,49 & 2,41 & 100 \\
\hline
\end{tabular}


Al cuestionarlos sobre si la organización patrocina los eventos realizados por la Coordinación de Pasantías de la Universidad (ítem 2), la totalidad de las empresas que formaron parte del estudio indica que nunca patrocinan tales eventos. En referencia al apoyo el desarrollo de proyectos comunes entre la universidad y la empresa (ítem 3 ), se observa que la relación entre la universidad y el sector productivo regional es casi nulo, según lo afirmado por el $95,18 \%$ de los representantes de estas organizaciones.

Por otra parte, al evaluar si las empresas otorgan algún premio o reconocimiento a los pasantes que realizan una labor destacada (ítem 4), se deduce que son poco dadas a otorgar algún tipo de distinción incluso si el alumno pasante ha cumplido una labor destacada por la que lo merezca. En efecto, el $79,51 \%$ señalan las opciones a veces o nunca. En esta misma dirección también se indagó acerca de si la empresa ofrece una oportunidad de trabajo a los pasantes de la Universidad al término de sus compromisos académicos (ítem 5). El 51,81\% de los encuestados expresa que nunca se ofrecer tal oportunidad de trabajo, seguido de un $45,18 \%$ que señala hacerlo algunas veces.

En torno al hecho de si la empresa ofrece becas o créditos a los pasantes para la culminación de sus estudios (ítem 6), es preciso señalar que las empresas donde los pasantes desarrollan sus prácticas profesionales no están obligadas a retribuir con asignaciones monetarias el trabajo de los estudiantes. Aun así, sería interesante que la Coordinación de Pasantías de la UNEFA - Táchira, en conjunto con las autoridades del Núcleo, establezcan y estrechen relaciones positivas con empresas, tanto del sector público como del privado, que contemplen algún tipo de incentivo para los estudiantes que cumplen su periodo de pasantías.

Por otra parte, al considerar si la organización ofrece cupos a los estudiantes de la Universidad para el desarrollo de sus pasantías industriales (ítem 7), los resultados evidencian que solo un $25,90 \%$ de las empresas consultadas ofrece siempre tales cupos a los estudiantes, seguido de un $50,60 \%$ que lo hace algunas veces.

Finalmente, solo un $16,27 \%$ de las empresas elegidas para el estudio siempre ofrece algún tipo de bonificación o pago a los estudiantes durante el desarrollo de las pasantías industriales, mientras 43,37\% lo hace a veces (ítem 8).

En relación a si las empresas mantienen comunicación con la Coordinación de Pasantías acerca de las oportunidades para los estudiantes de la Universidad (ítem 9), el 74,10\% señala que nunca mantiene este tipo de comunicación, seguido de un $23,49 \%$ que indica hacerlo algunas veces.

\subsection{Análisis de los resultados obtenidos a través del cuestionario aplicado a los estudiantes}

El cuadro 5 muestra algunos indicadores relaciones con la presencia o ausencia de ciertos beneficios que reciben los estudiantes por parte de las empresas en distribuciones de frecuencias de los 5 ítems dicotómicos que conforman esta escala.

Cuadro 5

Distribución de frecuencias por ítems de la primera parte del cuestionario aplicado a los estudiantes

\begin{tabular}{|c|c|c|c|c|}
\hline Ítem & & sí & no & Total \\
\hline \multirow[t]{2}{*}{1 Reconocimiento empresarial a los estudiantes por la labor realizada } & $\mathrm{f}$ & 64.00 & 124.00 & 188.00 \\
\hline & $\%$ & 34,04 & 65,96 & 100.00 \\
\hline \multirow[t]{2}{*}{2 Ofrecimiento de oportunidad laboral en la empresa } & $\mathrm{f}$ & 87.00 & 101.00 & 188.00 \\
\hline & $\%$ & 46,28 & 53,72 & 100.00 \\
\hline \multirow[t]{2}{*}{3 Ofrecimiento de beca o crédito para la culminación de estudios } & $\mathrm{f}$ & 0.00 & 188.00 & 188.00 \\
\hline & $\%$ & 0.00 & 100.00 & 100.00 \\
\hline
\end{tabular}




\begin{tabular}{lcccc}
\hline 4 Ofrecimiento de cupo por parte de la empresa & $\mathrm{f}$ & 52.00 & 136.00 & 188.00 \\
& $\%$ & 27,66 & 72,34 & 100.00 \\
\hline 5 Retribución económica al pasante & $\mathrm{f}$ & 103.00 & 85.00 & 188.00 \\
& $\%$ & 54,79 & 45,21 & 100.00
\end{tabular}

Fuente: Autor, Proceso de investigación.

En relación con los tipos de premio o reconocimiento que perciben los pasantes por las labores desempeñadas durante las pasantías (ítem 1), el $65,96 \%$ señala no recibir ningún incentivo por parte de las empresas donde desarrollan dichas actividades. E1 53,72\% indica que no se les ofrecen ninguna oferta de trabajo al término de las pasantías, lo que trae como consecuencia que los pasantes no se esmeren en buscar empresas consolidadas y competitivas como posibles destinos para sus pasantías (ítem 2).

En referencia a si la empresa proporciona una beca o crédito para la culminación de sus estudios (ítem 3), la totalidad de los encuestados declara que tal apoyo económico es inexistente. El72,34\%, coincide en que no se les ofrece cupo para el desarrollo de sus pasantías industriales (ítem 4) .Esta situación se agrava cuando el pasante se ve obligado a buscar una empresa para hacer sus pasantías, surgiendo casos donde los patronos usan los servicios de los jóvenes en lugar de ocupar al personal de la empresa. En relación al recibimiento de una retribución económica, 54,79\%) respondió que sí es retribuido; mientras que un 45,21\% señala que no. Las empresas que no pagan ningún emolumento a los pasantes lo hacen para no generar pasivos laborales; asimismo, argumentan que la oportunidad de estar en la empresa ya compensa el pago de la pasantía.

El cuadro 6 relaciona algunos indicadores referidos a las acciones que realizan los estudiantes de manera particular durante situaciones suscitadas a lo largo de la pasantía. Al valorar la experiencia que le proporcionan las pasantías para integrarse con éxito en el campo laboral (ítem 6) el 59,57\% responde estar totalmente de acuerdo. Esta cifra, sumada al 34,04\% que están medianamente de acuerdo, da idea de que el proceso de pasantías es una etapa muy bien considerada por los estudiantes como parte de su formación académica y profesional para la incorporación al mundo laboral, dado que permite demostrar las habilidades y destrezas para desarrollar las competencias en el área de su formación.

Al preguntarles a los estudiantes si las pasantías le han permitido desarrollar la confianza necesaria para desempeñarse en el ámbito de su profesión (ítem 7), casi la totalidad de los encuestados respondieron estar en total acuerdo. En efecto, así lo expresa el 96\% del conjunto.

En relación con si las actividades que se desarrollan durante las pasantías están directamente relacionadas con el perfil de la carrera, (ítem 8) el $55,85 \%$ está totalmente de acuerdo con que sí hay correspondencia, mientras que el 37,77\% están medianamente de acuerdo. Esto tiene que ver con el hecho de que en algunas empresas se les encomiendan actividades que no son propias del perfil en que se ha venido preparando del pasante, situación que desmotiva y ocasiona un impacto negativo a nivel personal por cuanto el estudiante no adquiere la experiencia laboral que necesita para incorporarse al mercado laboral.

Por otra parte, al considerar si las pasantías le han permitido desarrollar habilidades y destrezas necesarias para el desempeño profesional (Ítem 9 ), el $61,17 \%$ de los encuestados afirma estar totalmente de acuerdo con que el aserto, aparte de les ha facilitado la posibilidad de recibir ofertas en el mercado laboral. Desarrollar las competencias laborales tales como conocimientos, habilidades $\mathrm{y}$ actitudes, se traducen en resultados efectivos que contribuyen al logro de los objetivos de una organización. 
Cuadro 6

Distribución de frecuencias por ítems de la segunda parte del cuestionario aplicado a los estudiantes

\begin{tabular}{|c|c|c|c|c|c|c|c|}
\hline Ítem & & $T D$ & $M D$ & IN & MA & $T A$ & Total \\
\hline \multirow[t]{2}{*}{6 Integración exitosa al campo laboral } & $\mathrm{f}$ & 0 & 3 & 9 & 64 & 112 & 188 \\
\hline & $\%$ & 0 & 1,60 & 4,79 & 34,04 & 59,57 & 100 \\
\hline \multirow[t]{2}{*}{7 Desarrollo de la confianza como futuro profesional } & $\mathrm{f}$ & 0 & 3 & 3 & 55 & 127 & 188 \\
\hline & $\%$ & 0 & 1,60 & 1,60 & 29,26 & 67,55 & 100 \\
\hline \multirow[t]{2}{*}{8 Relación de las actividades realizadas con la carrera estudiada } & $\mathrm{f}$ & 0 & 7 & 5 & 71 & 105 & 188 \\
\hline & $\%$ & 0 & 3,72 & 2,66 & 37,77 & 55,85 & 100 \\
\hline \multirow[t]{2}{*}{9 Desarrollo de habilidades y destrezas necesarias para la profesión } & $\mathrm{f}$ & 1 & 8 & 5 & 59 & 115 & 188 \\
\hline & $\%$ & 0,53 & 4,26 & 2,66 & 31,38 & 61,17 & 100 \\
\hline \multirow[t]{2}{*}{10 Reconocimiento al desempeño del estudiante } & $\mathrm{f}$ & 2 & 81 & 33 & 22 & 50 & 188 \\
\hline & $\%$ & 1,06 & 43,09 & 17,55 & 11,70 & 26,60 & 100 \\
\hline \multirow[t]{2}{*}{11 Pasantías obtenidas por diligencia de la universidad } & $\mathrm{f}$ & 17 & 112 & 6 & 52 & 1 & 188 \\
\hline & $\%$ & 9,04 & 59,57 & 3,19 & 27,66 & 0,53 & 100 \\
\hline \multirow[t]{2}{*}{12 Efectividad de los procedimientos administrativos } & f & 12 & 79 & 19 & 52 & 26 & 188 \\
\hline & $\%$ & 6,38 & 42,02 & 10,11 & 27,66 & 13,83 & 100 \\
\hline \multirow[t]{2}{*}{ 13. Resolución de eventualidades } & $\mathrm{f}$ & 21 & 40 & 19 & 67 & 41 & 188 \\
\hline & $\%$ & 11,17 & 21,28 & 10,11 & 35,64 & 21,81 & 100 \\
\hline \multirow[t]{2}{*}{$\begin{array}{l}14 \text { Cupo tramitado a través de la cooperación universidad-sector } \\
\text { productivo }\end{array}$} & $\mathrm{f}$ & 82 & 55 & 8 & 23 & 20 & 188 \\
\hline & $\%$ & 43,62 & 29,26 & 4,26 & 12,23 & 10,64 & 100 \\
\hline \multirow[t]{2}{*}{15 Receptividad por parte de la empresa } & $\mathrm{f}$ & 9 & 28 & 6 & 100 & 45 & 188 \\
\hline & $\%$ & 4,79 & 14,89 & 3,19 & 53,19 & 23,94 & 100 \\
\hline \multirow[t]{2}{*}{16 Trámite personal de la pasantía } & $\mathrm{f}$ & 6 & 9 & 4 & 89 & 80 & 188 \\
\hline & $\%$ & 3,19 & 4,79 & 2,13 & 47,34 & 42,55 & 100 \\
\hline \multirow[t]{2}{*}{17 Apoyo efectivo del tutor académico } & $\mathrm{f}$ & 4 & 47 & 1 & 67 & 69 & 188 \\
\hline & $\%$ & 2,13 & 25,00 & 0,53 & 35,64 & 36,70 & 100 \\
\hline \multirow[t]{2}{*}{18 Disponibilidad de tiempo, por parte del tutor académico } & $\mathrm{f}$ & 25 & 96 & 10 & 37 & 20 & 188 \\
\hline & $\%$ & 13,30 & 51,06 & 5,32 & 19,68 & 10,64 & 100 \\
\hline
\end{tabular}

Fuente: Autor, Proceso de investigación.

Al indicador que evalúa si la empresa reconoce que su labor es altamente calificada para un buen desempeño profesional (ítem 10), el 43,09\% los estudiantes encuestados manifiestan estar medianamente en desacuerdo con el reconocimiento de la empresa. Por lo general, se menosprecia el talento de los profesionales noveles para asumir responsabilidades y se prefiere la experiencia laboral.
En relación con la consideración de si el proceso para la obtención de las pasantías fue debidamente diligenciado por el personal de la Universidad (ítem 11 ), el 59,57\% manifiesta estar muy en desacuerdo. Por otra parte, al cuestionarles si los procedimientos administrativos en la universidad facilitaron al estudiante la obtención de las pasantías (ítem 12) el $42,02 \%$ señala estar medianamente en desacuerdo con este hecho. 
También se preguntó a los estudiantes acerca de su capacidad para resolver eventualidades durante el proceso de pasantías a través de la Coordinación de la Universidad (ítem 13). 34,64\% expresa que le resultó medianamente fácil solventar los problemas a través de la Coordinación de Pasantías. En referencia a si el cupo para el desarrollo de la pasantía fue tramitado a través de la cooperación que la universidad mantiene con el sector productivo (ítem 14) el 43,62\% manifestó estar en total desacuerdo, lo que indica que la relación sector productivouniversidades es precaria y constituye un elemento obstaculizador para el desarrollo de las pasantías y de las posibilidades de entrar al mundo laboral.

Al evaluar si la empresa se mostró receptiva al momento de presentarse con la carta de postulación como pasante (ítem 15), el 53,20\% de los encuestados indica su total acuerdo con que la empresa se mostró proclive a su recibimiento, dando seguridad al momento de presentarse en la organización. En ese sentido, se les cuestionó si tramitó personalmente el cupo para el desarrollo de su pasantía industrial (ítem 16). Prácticamente la totalidad de los encuestados señalaron mediante la opción totalmente de acuerdo que la tramitación se cumplió por cuenta propia $(47,34 \%$ totalmente de acuerdo y $42,55 \%$ de acuerdo).

En referencia al apoyo del tutor académico y su ayuda para solventar cualquier dificultad que surgiera durante el desenvolvimiento en la empresa (ítem 17), el 36,70\% manifestó su total acuerdo, a lo que se suma el $35,64 \%$ que se encuentra medianamente de acuerdo con que el tutor académico ha representado un apoyo durante las pasantías. En esa misma dirección se indagó si el tutor académico posee la disponibilidad de tiempo necesaria para el asesoramiento (ítem 18). El 51,10\% respondió que está en medianamente en desacuerdo. Ello ocurre, entre otras razones, porque la UNUFA no contrata tutores, por lo que recurre a profesores de planta.

\section{Conclusiones}

Una vez realizado el análisis sobre la temática de estudio referente al fortalecimiento de la cooperación académica entre la Universidad Nacional Experimental Politécnica de la Fuerza Armada Bolivariana (UNEFA - Táchira) y el sector productivo regional durante el proceso de las pasantías universitarias de los estudiantes que están por finalizar sus carreras se destacan las siguientes conclusiones:

En cuanto a la vinculación académica existente entre la UNEFA - Táchira y el sector productivo regional, los estudiantes, tutores y empresas sostienen que a pesar de que la universidad realiza acercamientos importantes con el sector productivo a fin de ubicar los pasantes, existe aún poca comunicación, ausencia de alianzas estratégicas para patrocinar eventos académicos y una marcada carencia de incentivos por parte del sector productivo. Estos hallazgos son un indicativo de que la cooperación académica se limita estrictamente a las pasantías profesionales y la asociación se basa principalmente en el suministro de capital humano estudiantil, lo que termina por generar distanciamiento entre los organismos que obstaculizan los cambios necesarios en el tema de la cooperación académica.

Por otro lado, se tiene que el proceso de pasantías llevado a cabo en la UNEFA -Táchira es percibido como desfavorable por los pasantes y los tutores. Ante esta situación, surge la necesidad de reforzar la Coordinación de Pasantías- UNEFA, bien con la incorporación de personal que ayude atender de manera eficiente a los estudiantes de todos los turnos, bien ofreciendo incentivos a los tutores a fin de que tengan una partición más activa.

En relación con los factores que obstaculizan la cooperación académica entre la UNEFA - Táchira y el sector productivo, se tiene que existen variables internas que limitan el buen desarrollo de las pasantías. Entre ellas se pueden mencionar que la Coordinación de Pasantías no cuenta con el personal suficiente o que existen barreras motivacionales con los tutores dada la ausencia de incentivos y reconocimiento, lo cual degenera en desinterés por la realización de esa labor. También existen factores externos, tales como el desinterés del entorno 
productivo por la capacitación, la transferencia de información y el ofrecimiento de estímulos económicos a los pasantes. Una vez reconocidas estas limitantes se hace necesario establecer instrumentos de promoción a fin de superar las barreras en favor de la cooperación académica.

La institución universitaria podría potenciar la vinculación con organismos externos a través de una Unidad de Pasantías que promueva una cultura universitaria que favorezca el entrenamiento dentro de las empresas como una propuesta académica que geste el desarrollo del conocimiento acorde a los requerimientos del dinámico mercado laboral. Optimizar la integración académica en el proceso de las pasantías universitarias es un objetivo primordial, pero supone el diseño de estrategias que ubiquen a los pasantes y tutores empresariales como principal foco de atención y creen las condiciones idóneas a fin de mejorar las relaciones entre las diversas instituciones en estudio.

El avance de la cooperación académica que se está dando en otras instituciones hace que las pasantías resulten una alternativa real y prioritaria en la UNEFA - Táchira, a fin de fortalecer la gestión del conocimiento de los futuros profesionales. A su vez, la cooperación académica constituye un motor que impulsa la cooperación con el sector productivo y una oportunidad para lograr el desarrollo institucional, las alianzas estratégicas y, en general, la cultura de trabajo colectivo para todos los involucrados.

\section{Referencias}

Acevedo, J; González, E. (2017). Concepciones sobre las prácticas evaluativas entre docentes de programas universitarios de enfermería. Perspectivas 2 (1). 65-77

AlfiaGazizova, E. K. (2015). Academic-Industry Partnership in Russia and Abroad. Procedia Social and BehavioralSciences, 214(5), 941945.

Allende Hernández, O.; GonzalezCarella, M. I. y Zanfrillo, A. I. (2010). Un estudio de estrategias de vinculación universidad y entorno socioproductivo. Temas de ciencia y tecnología, 14(42), $41-52$.

Corti, A. M.; Oliva, D. L. y De la Cruz, S. (2015). La internacionalización y el mercado universitario. Revista de la educación superior, XLIV-2(174), 47-60.

Dan, C. D. (2013). Why Should University and Business Cooperate? A Discussion of Advantages and Disadvantages. International Journal of Economic Practices and Theories, 3(1), 67-74.

De La Vega, A. y Arakaki, M. (2011). Las prácticas pre profesionales en la formación en Ciencias de la Información: el caso de la Pontificia Universidad Católica del Perú (PUCP). Revista interamericana de bibliotecología, 34(1), 77-86.

García Palma, J. J. (2013). Movilidad estudiantil internacional y cooperación educativa en el nivel superior de educación. revista iberoamericana de educación, 61, 59-76.

Grotta, A. y McGrath, D. (2013). The Role of Internships in Raising Undergraduates' Awareness and Perception of Extension. Journal of Extension, 51(4), 1-7.

Hernández Sampieri, R., Fernández Collado, C. y Baptista Lucio, P. (2006). Metodología de la investigación. México D.F:: McGraw-Hill.

Kneller, R. (2014). University-industry cooperation and technology transfer in Japan compared with the United States: Another reason for Japan's economic Malaise? Journal of International Law, 24(2), 329-449.

Maculan, A. M. y Carvalho de Mello, J. M. (2009). University start-ups for breaking lock-ins of the Brazilian economy. Science and Public Policy, 36(2), 109-114.

Mgaya, K. y Mbekomize, C. (2014). Benefits to host organizations from participating in internship programs in Botswana. Asia-Pacific Journal of Cooperative Education, 15(2), 129-144.

Miranda, E. M. y Salto, D. D. (2012). Cooperación académica internacional: entre la política y la gestión universitaria. Revista argentina de educación superior, 4(4), 34-52.

Morales Rubiano, M. E.; Sanabria Rangel, P. E.; Plata Pacheco, P. A. y Ninco Hernández, F. A. (2015). Research Results Transfer towards the 
Productive Sector via Research Collaboration in Four Colombian Public Universities. Journal of Technology Management \& Innovation, 10(4), 28-44.

Nicholas, A. J. (2016). Internships: Experiential Learning,Academic Connection andAssessment. Faculty and Staff - Articles \& Papers, Paper 61.

Rikap, C. (2012). The connection between the university and the productive sector: technological transference. Ecos de Economía: A Latin American Journal of Applied Economics, 16(34), 127-149.

Renganathan, S.; Abdul Karim, Z. A. B. y Li, C. S. (2012). Students' perception of industrial internship programme. Education + Training, 54(2/3), 180-191.

Rigsby, J. T.; Addy, N.; Dean, C. H. y Polledo, D. (2013). An examination of internships and job opportunities. The Journal of Applied Business Research, 29(4), 1131-1144.

SampaioCorrêa da Silva, C. y Pereira Teixeira, M. A. (2013). Internship Experiences: Contributions to the School-to-Work Transition. Paidéia, 23(54), 103-112.

Solanas, F. (2014). Intercambio cooperativo versus mercantilización competitiva: las políticas de movilidad académica en el mercosur y la Unión Europea. Revista iberoamericana de educación superior, V(12), 3-22.

Templeton, W. K.; Updyke, K. A. y Bennett, R. B. (2012). Internships and the Assessment of Student Learning. Scholarship and Professional Work - Business, 4(2), 26-38.

Weible, R. (2009). Are Universities Reaping the Available Benefits InternshipPrograms Offer? Journal of Education for Business, 85(2), 59-63. 